\title{
ROLE OF LUNG ULTRASOUND IN DIAGNOSIS AND FOLLOW UP OF PNEUMONIA IN ADULTS: IS IT SIGNIFICANT?
}

\author{
By
Ahmed Mohammed Fekry El-Deek, El-Sayed Mohamed Abd El-Hamid Hassan and Ramadan Shawky*

Departments of Radio diagnosis \& Diagnostic Radiology and Chest*, Faculty of Medicine, Al-Azhar University, New Damietta, Egypt

E-mail: $\underline{\text { deekscan@yahoo.com }}$

\begin{abstract}
Background: Pneumonia is a common and serious infectious disease that can cause high mortality. Lung ultrasound (LUS) is increasingly utilized in emergency and critical settings. The role of LUS in evaluation of pneumonia is becoming more and more important.

Objective: The current study was performed to compare the diagnostic accuracy of lung ultrasound against other modalities for evaluation and follow up of pneumonia in adult patients.

Patients and Methods: One hundred and eight patients (70 males, 38 females) aged $57.33 \pm 9.39$ years admitted to the Chest Department with pneumonia from March to November 2019. After institute ethical committee clearance and written informed consent, each participant underwent chest X-ray (CXR), LUS and computed tomography (CT) within 6 hours from admission by 2 different radiologists being blind to the results of the other examination to minimize the bias. Follow up US was done after adequate medical treatment (7-14 days) to detect its ability for following the patients up.

Results: A total of 108 patients who fulfilled the eligibility criteria were enrolled in this study LUS showed positive findings in $101(93.5 \%)$ patients in the form of isoechoic area in $6(5.5 \%)$ patients, dynamic air bronchogram alone in $25(23.1 \%)$ patients and the last one was associated with all other sonographic signs of the studied patients. During follow up period, 55 (54.5\%) of cases showed total resolution, while 33 cases $(32.7 \%)$ showed regressive course of their sizes. Ten cases $(9.9 \%)$ showed rather stationary course, while three cases $(2.9 \%)$ showed progression course. The associated pleural effusion was resolved in 36 cases (90\%) out of 40. Compared to CT, LUS showed a sensitivity and specificity of $93.33 \%$ and $94.74 \%$ for pneumonia detection respectively with $95.4 \% \mathrm{PPV}, 92.4 \% \mathrm{NPV}$, and $93.91 \%$ accuracy.
\end{abstract}

Conclusion: LUS considered being a good diagnostic and following up tool when pneumonia is suspected, its results were closer to $\mathrm{CT}$ with minimal cost.

Key Words: Chest X-Ray, Computerized tomography, Lung ultrasound, Pneumonia.

\section{INTRODUCTION}

Pneumonia is a major health problem worldwide, failure of early detection and distribution of treatment may lead to significant morbidity and mortality (Yang et al., 2016).
Absence of specific guidelines for pneumonia diagnosis means that its diagnosis is usually based on clinical signs, symptoms, history-taking, and physical examination (Caiulo et al., 2013). Chest X-ray (CXR) is well known as an essential tool in pneumonia 
diagnosis, but it has low sensitivity and specificity as well as it is associated with considerable practical delays related to processing (Blaivas, 2012).

Although computed tomography (CT) has been considered as the "gold standard" technique in the diagnosis of pneumonia during the last decade, it can't be used as the first-line for radiological evaluation in all patients with suspected pneumonia due to the high radiation and the fact of being pricey and unavailable in some places. Especially ionizing radiations which magnify the risk of gene mutations and cancer evolution (Rennis et al., 2017).

Lung ultrasound (LUS) was promoted as a modality that can overcome many of the above-mentioned limitations of other tools in the diagnosis of pneumonia in multiple settings (Lichtenstein, 2009). However, few studies were concerned about its usage in the diagnosis and follow up of pneumonia.

The current study was performed to assess the role of LUS in the diagnosis and follow-up of pneumonia compared to $\mathrm{CT}$ in adult population.

\section{PATIENTS AND METHODS}

This study was a prospective cross sectional randomized study which was conducted in Chest and Radiology Departments from March to November 2019 on 115 patients presented with respiratory distress and suspected to have pneumonia on the basis of clinical examination and chest radiography. Institutional research board approval had been gained, and all patients had assigned informed consents prior to study processing.
Enrolled patients underwent both chest US and chest CT, and 108 patients had final diagnosis of pneumonia and they were included in this study. Other seven patients with uncertain diagnoses were excluded. Pregnant women were also excluded because of the restrictions in the use of CT chest which is required in the study.

All patients underwent demographic data collection, complete history taking, thorough general and local chest examination, routine laboratory investigation, CXR, CT chest scan, and transthoracic LUS.

Chest radiography was performed using Toshiba diagnostic equipment (Toshiba Medical Systems, Japan) by posterior-anterior and lateral views in the upright patients and anterior-posterior view in the supine patients, following standardized hospital diagnostic protocol. The film was digitally reviewed firstly blinded to the results of LUS and CT. CXR was considered positive when at least one typical consolidation was visualized.

A low-dose HRCT scan was performed on admission using multi slice CT scan (Cortellaro et al., 2012). Chest CT was performed by Toshiba aquilion prime 80-dual MDCT (Toshiba Medical Systems, Japan). CT of the chest was performed on admission as the gold standard for diagnosis.

LUS was performed using $3 \mathrm{MHz}$ Curvilinear Transducer of Voluson E6 (GE, Germany) with a $3 \mathrm{MHz}$ convex transducer and was targeted to evaluate lung consolidations with the morphologic characteristics of pneumonia. Patients were examined anteriorly in a supine 
position and the posterior areas were studied in the lateral decubitus or sitting position according to clinical status. Each hemithorax was divided into five areas, two anterior, two lateral, and one posterior, for a total of 10 areas bilaterally. The anterior chest wall was marked off from the parasternal line to the anterior axillary line. This zone was split into an upper region (from the collar bone to the second intercostal space) and a lower region (from the third intercostal space to the diaphragm). The lateral area (anterior to posterior axillary line) was split into upper and lower halves. Finally, the posterior area was identified from the posterior axillary line to the paravertebral line. The US transducer was moved until a rib interspace was located. The probe was then panned horizontally and vertically to the extent possible to allow the broadest sweep through the area being imaged. Raising the arm above the head increases the rib space distance and facilitates scanning. Scanning was performed during quiet respiration, to allow for assessment of normal lung movement, and, in suspended respiration, when a lesion can be examined in detail. The echogenicity of a lesion was compared with that of the liver and characterized as hypoechoic, isoechoic, or hyperechoic.

LUS and CT images were examined for the presence of parenchymal consolidation, lung necrosis, abscess, pleural effusion and the presence of loculation or fibrin strands within the pleural fluid. LUS and CT were performed by one of the investigators who participated in the study. The investigators were intensivists with at least 5 years' experience on ultrasonography.A radiologist reported the US findings blindly to the results of the CT.

Statistically analysis was performed using SPSS software version 23 for Windows (SPSS Inc., Chicago, IL, USA). Quantitative data were expressed as the mean $\pm S D$, and qualitative data were expressed as absolute frequencies 'number' and relative frequencies (percentage). Percent of categorical variables was compared using the Chi Square-test when appropriate. Sensitivities, specificities, positive likelihood ratio and negative likelihood ratio with their respective $95 \%$ confidence intervals were calculated. P-value less than 0.05 were considered statistically significant.

\section{RESULTS}

A total of 108 patients who fulfilled the eligibility criteria were enrolled in the current study. They were 38 females $(35.2 \%)$ and 70 males $(64.8 \%)$ with their mean age of $57.33 \pm 9.39$ years (range
22.0-58.0). The patient's clinical characteristics regard to blood pressure, pulse, temperature, respiratory rate and level of consciousness (Table 1). 
606

AHMED MOHAMMED FEKRY EL-DEEK et al.,

Table (1): Demographic data and clinical assessment in the study group $(n=108)$

\begin{tabular}{|l|c|}
\hline Parameters & Values \\
\hline Age (years) & $57.33 \pm 9.39$ \\
\hline Sex (no; $)$ & \\
Male & $70(64.8 \%)$ \\
Female & $38(35.2 \%)$ \\
\hline Systolic blood pressure $(\mathrm{mmgH})$ & $98.21 \pm 15.75$ \\
\hline Diastolic blood pressure $(\mathrm{mmgH})$ & $63.88 \pm 13.45$ \\
\hline Pulse (rate/min) & $98.55 \pm 9.95$ \\
\hline Temperature $\left({ }^{\circ} \mathrm{C}\right)$ & $38.73 \pm 0.75$ \\
\hline Respiratory rate $($ rate/min) & $34.42 \pm 3.98$ \\
\hline Conscious level: & $13(12.0 \%)$ \\
Confused & $95(88.0 \%)$ \\
Normal &
\end{tabular}

Eighty -eight patients $(81.5 \%)$ were positive by CXR, ultrasound and CT (Figure 1), 101 patients $(93.5 \%)$ were positive by ultrasound and CT while 104 cases $(96.3 \%)$ positive by $\mathrm{CT}$. There was significant difference $(\mathrm{P}=0.014)$ between LUS and CXR in detecting pneumonia; pneumonia was detected in 101 patients $(93.5 \%)$ with LUS, whereas in 88 patients $(81.5 \%)$ with CXR. However, there was no significant difference $(\mathrm{P}=0.535)$ between LUS and chest $\mathrm{CT}$ in detecting pneumonia; 101 patients $(93.5 \%)$ had positive signs of pneumonia with LUS compared to 104 patients $(96.3 \%)$ of pneumonia detected by chest CT (Table 2).

Table (2): Comparison between CXR and LUS findings in detecting pneumonia

\begin{tabular}{|c|c|c|c|c|c|}
\hline Parameters & Findings & $\begin{array}{c}\text { CXR } \\
(\mathrm{N}=108) \\
{[\mathrm{n}(\%)]}\end{array}$ & $\begin{array}{c}\text { LUS } \\
(\mathrm{N}=108) \\
{[\mathrm{n}(\%)]}\end{array}$ & $\begin{array}{l}\text { Chi- } \\
\text { squar } \\
\text { e } \\
\end{array}$ & $\begin{array}{c}\mathrm{P} \\
\text { valu } \\
\mathrm{e} \\
\end{array}$ \\
\hline \multirow{2}{*}{ Signs of pneumonia } & Absent & $20(18.5 \%)$ & $7(6.5 \%)$ & \multirow{2}{*}{6.095} & \multirow{2}{*}{$\begin{array}{c}0.01 \\
4\end{array}$} \\
\hline & Present & $88(81.5 \%)$ & $101(93.5 \%)$ & & \\
\hline & & $\begin{array}{c}\text { LUS } \\
(\mathrm{N}=108) \\
{[\mathrm{n}(\%)]}\end{array}$ & $\begin{array}{c}\text { Chest CT } \\
(\mathrm{N}=108) \\
{[\mathrm{n}(\%)]}\end{array}$ & & \\
\hline \multirow{2}{*}{ Signsofpneumonia } & Absent & $7(6.5 \%)$ & $4(3.7 \%)$ & \multirow{2}{*}{0.383} & 0.53 \\
\hline & Present & $101(93.5 \%)$ & $104(96.3 \%)$ & & 6 \\
\hline
\end{tabular}

CXR, chest $\mathrm{x}$ ray; LUS, lung ultrasound;CT, computed tomography 


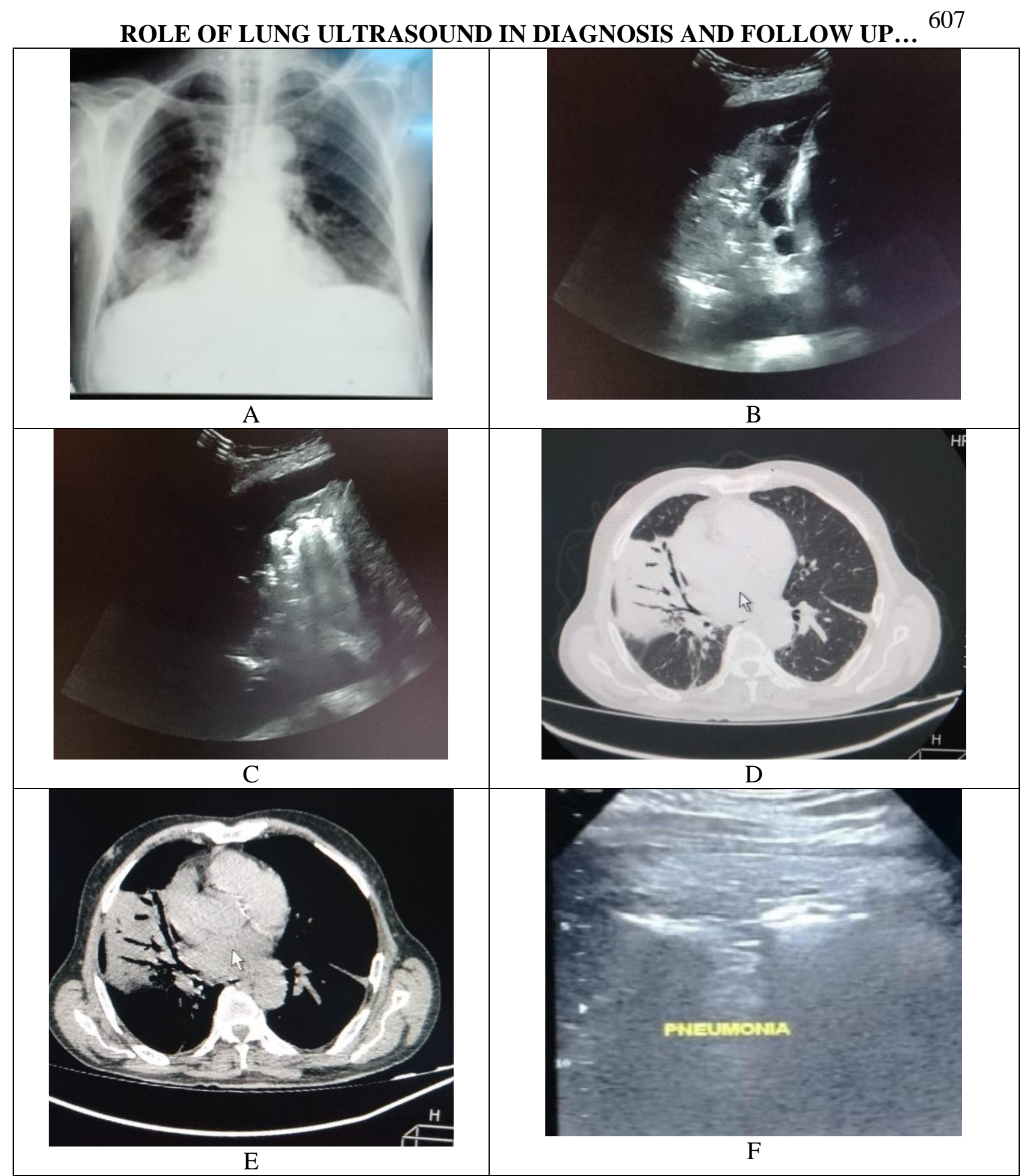

Figure (1): 36 years old male presented with acute pneumonia a) CXR PA view shows non homogeneous opacity involve right lower lung zone. b-c) LUS showing loss of A lines, subpleural consolidation with liver like echogenecity, poorly defined hypoechoic area with irregular outline, B lines, dynamic air bronchogram (noted during scanning), reduced or absent lung sliding (noted during scanning) and parapneumonic pleural effusion. d-e) Axial CT cuts in lung and mediastinal windows reveled pulmonary consolidation with air bronchogram, F) follow up Lung ultrasound revealed; some improvement of the pneumonic consolidation with no pleural effusion.

The LUS showed positive findings in 101 $(93.5 \%)$ patients in the form of isoechoic area in $6(5.5 \%)$ patients, dynamic air bronchogram alone in $25(23.1 \%)$ patients and the last one was associated with all other sonographic signs of the studied patients (Table 3). 
AHMED MOHAMMED FEKRY EL-DEEK et al.,

Table (3): LUS findings of pneumonia in the studied cases $(n=108)$

\begin{tabular}{|l|c|}
\hline LUS findings & $\mathrm{n}(\%)$ \\
\hline Negative & $\mathbf{7 ( 6 . 5 \% )}$ \\
\hline Positive & $\mathbf{1 0 1}(\mathbf{9 3 . 5 \% )}$ \\
\hline Dynamic air bronchogram & $25(23.1 \%)$ \\
\hline Dynamic air bronchogram and subpleural consolidation & $22(20.4 \%)$ \\
\hline Dynamic air bronchogram and fluid bronchogram & $14(13.0 \%)$ \\
\hline Dynamic air bronchogram and free pleural effusion & $11(10.2 \%)$ \\
\hline Dynamic air bronchogram and B-lines & $9(8.3 \%)$ \\
\hline Dynamic air bronchogram and complex septated pleural effusion & $7(6.5)$ \\
\hline Isoechoic area & $6(5.6 \%)$ \\
\hline Dynamic air bronchogram and complex nonseptated pleural effusion & $4(3.7 \%)$ \\
\hline Dynamic air bronchogram and hypoechoic area & $3(2.8 \%)$ \\
\hline
\end{tabular}

For the LUS positive (101 cases) who were subjected to follow up US after adequate treatment (within 7-14 days), 55 (54.5\%) cases out of the 101 positive cases showed almost total resolution of the pneumonic hepatization while 33 cases $(32.7 \%)$ showed regressive course of their sizes. Ten cases $(9.9 \%)$ showed rather stationary course while three cases (2.9\%) showed progression of the extension of the pneumonic hepatization. The associated pleural effusion was resolved in 36 cases $(90 \%)$ out of 40 while 4 cases showed regressive, stationary and progressive course (Table 4).

Table (4): Follow up results for LUS positive (103 cases)

\begin{tabular}{|l|c|c|c|c|}
\hline \multirow{2}{*}{ Follow up } & \multicolumn{2}{c|}{$\begin{array}{c}\text { Follow up US for } \\
\text { hepatization }\end{array}$} & \multicolumn{2}{c|}{$\begin{array}{c}\text { Follow up US for } \\
\text { pleural effusion }\end{array}$} \\
\cline { 2 - 5 } Parameters & No & $\%$ & No & $\%$ \\
\hline Total positive cases & 101 & 100 & 40 & 100 \\
\hline Complete resolution & 55 & 54.5 & 36 & 90 \\
\hline Regressive course & 33 & 32.7 & 2 & 5 \\
\hline Stationary course & 10 & 9.9 & 1 & 2.5 \\
\hline Progressive course & 3 & 3 & 1 & 2.5 \\
\hline
\end{tabular}

According to the above results, CXR showed in comparison to CT sensitivity and specificity of $83.81 \%$ and $95.79 \%$ for the detection of pneumonia respectively, with 95.9\% PPV, 83.4\% NPV and $81.42 \%$ accuracy.
LUS showed in comparison to CT sensitivity and specificity of $93.33 \%$ and $94.74 \%$ for the detection of pneumonia respectively with 95.4\% PPV, 92.4\% NPV and 93.91\% accuracy (Table 5).

Table (5): The diagnostic parameters of CXR and LUS compared to chest CT for the diagnosis of pneumonia

\begin{tabular}{|l|c|c|}
\hline Parameters & CXR & LUS \\
\hline Sensitivity & $83.81 \%$ & $93.33 \%$ \\
\hline (95\% CI) & $(75.3-90.3)$ & $(86.7-97.3)$ \\
\hline Specificity & $95.79 \%$ & $94.74 \%$ \\
\hline (95\% CI) & $(89.6-98.8)$ & $(88.1-98.2)$ \\
\hline PPV & $95.9 \%$ & $95.4 \%$ \\
\hline NPV & $83.4 \%$ & $92.4 \%$ \\
\hline Accuracy & 81.42 & $93.91 \%$ \\
\hline (95\% CI) & $77.41-87.36$ & $87.86-97.52$ \\
\hline
\end{tabular}

CI: Confidence interval, PPV: Positive predictive value, NPV: Negative predictive value, 


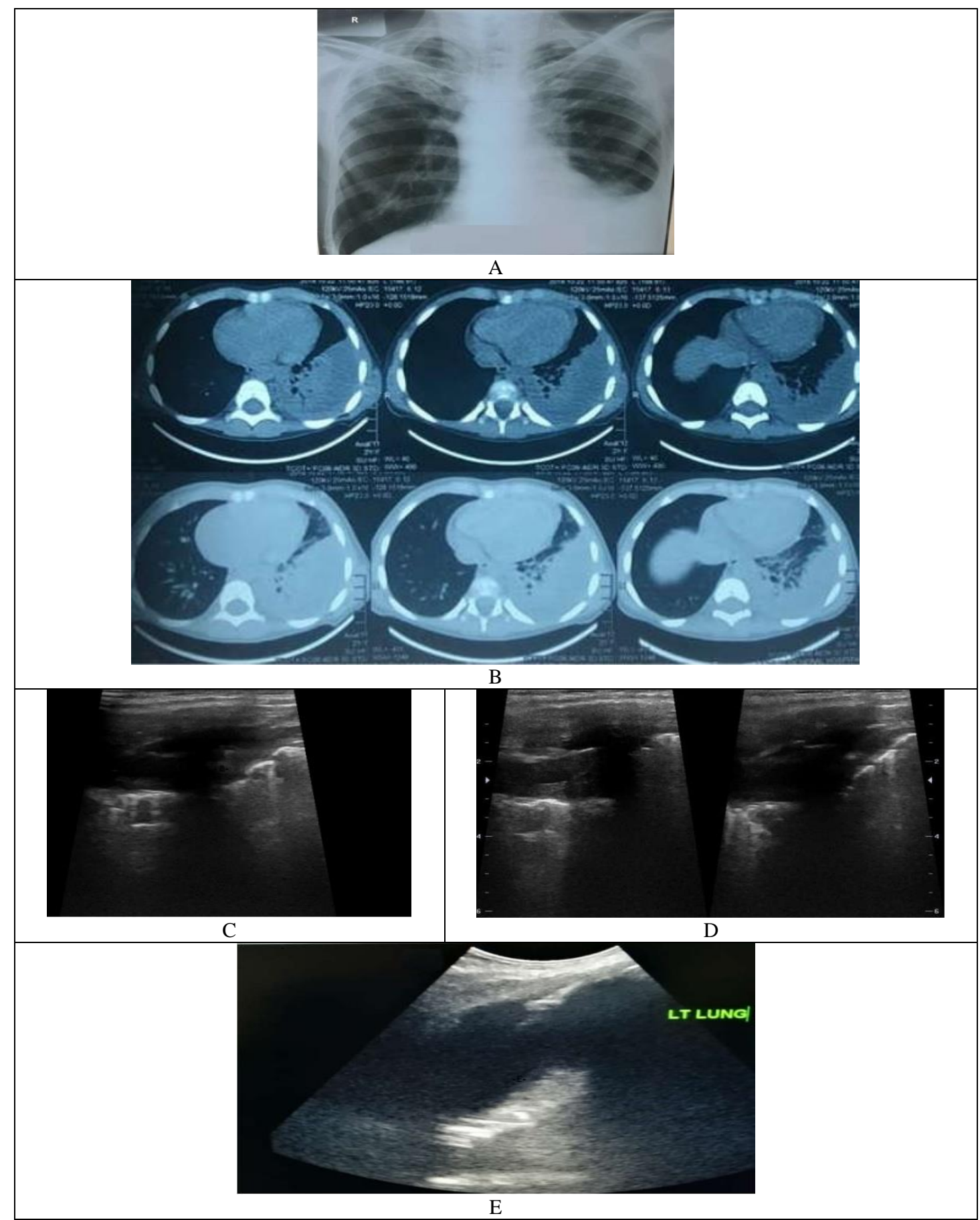

Figure (2): A 38 years old male patient complaining of fever, cough and RD; A) Chest $x$ ray PA view; non homogenous opacity involve $L T$ lower lung zone, with obliterated LT costophrenic angle by homogenous opacity devoid of lung marking, rising laterally to the LT axilla. B) Non contrast CT chest in lung and Mediastinal windows: consolidation collapse involve LT lower lung lobe, mild LT side pleural effusion. C and D) Lung US: loss of A lines, poorly defined hypoechoic area with irregular outline, solid non aerated airless lung with scalloped pleural borders, mild parapneumonic pleural effusion. E) Follow up by Lung US: shows only mild LT side clear free pleural effusion. 


\section{DISCUSSION}

In our study, LUS was as reliable as $\mathrm{CT}$ in detecting cases of pneumonia and in following up positive cases. LUS can substantially decrease the practical delays associated with CXR and avoiding radiation exposure with CT (Rennis et al., 2017). LUS showed positive findings in $93.5 \%$ of patients in the form of isoechoic area in $5.5 \%$ of patients; dynamic air bronchogram alone in $23.1 \%$ of patients and the last one was associated with all other sonographic signs of the studied patients. In agreement with our findings, Parlamento et al. (2009), air bronchogram, was found in $68.8 \%$ patients with confirmed pneumonia diagnosis; $50 \%$ of the patients with confirmed pneumonia presented with Blines and dynamic air bronchograms, whereas pleural effusion was found in $34.4 \%$ patients.

Moghawri et al. (2017) reported that LUS showed positive findings in $96.7 \%$ patients in the form of dynamic air bronchogram alone in $19.2 \%$ patients, and was associated with all other sonographic signs of the studied patients.

The results of the present study were partially in agreement with those of Cortellaro et al. (2012) who stated that pneumonia appeared as a pattern of consolidation with dynamic air bronchogram in $91.3 \%$ of patients, and alveolointerstitial syndrome in $52.5 \%$. Pleural effusion was present in $39 \%$ of patients with final diagnosis of pneumonia and in $15 \%$ of patients without pneumonia, confirming it to be a nonspecific sign.

Agmy and Ahmed (2013) performed LUS, CXR, and CT scan on patients presented to the emergency department with suspected pneumonia; air bronchogram was found in $82 \%$ patients with confirmed pneumonia. Alkhayat and Alam-Eldeen (2014) stated that air bronchogram was seen in $87 \%$ of patients and blurred margins, but pleural effusion were present in 54\%. This discrepancy with the current study may be attributed to the selection of patients with early pneumonia based on the early clinical data.

During follow up of LUS positive, $54.5 \%$ of cases showed total resolution of the pneumonic hepatization, while $32.7 \%$ showed regressive course of their sizes, 9.9\% showed rather stationary course, while $2.9 \%$ showed progression of the extension of the pneumonic hepatization. The associated pleural effusion was resolved in $90 \%$, while $10 \%$ of cases showed regressive, stationary and progressive course. These results were in agreement with Saraya and El Bakry (2017) concluded that US could be considered as a good diagnostic and follow up tool when pneumonia especially in pediatric age group.

In the current study, CXR showed in comparison to $\mathrm{CT}$ sensitivity and specificity of $83.81 \%$ and $95.79 \%$ for the detection of pneumonia respectively with 95.9\% PPV, 83.4\% NPV and $81.42 \%$ accuracy. LUS showed in comparison to CT sensitivity and specificity of $93.33 \%$ and $94.74 \%$ for the detection of pneumonia respectively with $95.4 \%$ PPV, 92.4\% NPV and $93.91 \%$ accuracy. These results were in agreement with Andrea et al. (2016) where LUS maintained a high diagnostic accuracy compared to $\mathrm{CT}$, but CXR did not. 
In agreement with Moghawri et al. (2017) where pneumonia was detected in 96.7\% patients by LUS. However, there was no significant difference between LUS and chest CT in detecting pneumonia. $96.7 \%$ of patients had positive signs of pneumonia with LUS as well as chest CT. Moreover, LUS had a sensitivity and positive predictive value of $97.4 \%$, specificity of $25 \%$, and accuracy of $95 \%$ in the detection of pneumonia. Also, Saraya and El Bakry (2017) found that, compared to CT, ultrasound showed a sensitivity and specificity of $72.2 \%$ and 95\% for pneumonia detection respectively with $96.3 \%$ PPV, 5\% NPV, 3.7\% FDR and $80.3 \%$ accuracy.

Our data were partially concordant with those of Cortellaro et al. (2012) in which the sensitivity of LUS was $96 \%$, whereas that of CXR was $69 \%$ of patients.

Agmy and Ahmed (2013) reported similar results, where the sensitivity of LUS was 97\%. Parlamento et al. (2009) also reported that sensitivity of LUS was $96 \%$, whereas that of CXR was $69 \%$. LUS by Saraya and his colleagues (2017) showed in comparison to CT sensitivity and specificity of $72.2 \%$ and $95 \%$ for the detection of pneumonia respectively with 96.3\% PPV, 5\% NPV, 3.7\% FDR and $80.3 \%$ accuracy.

CXR by Fahmy and Kinawy (2018) showed false-negative examination with no abnormal findings in $18.75 \%$ patients and false positive examination in $6.2 \%$ patients. The sensitivity was $81.25 \%$ (95\% CI $73.8-95.6 \%$ ), while LUS was falsely positive in two cases $(6.2 \%)$ and false negative in two patients $(6.2 \%)$. The sensitivity and the specificity of LUS were
$87.5 \%$ (95\% CI $78.9-92.7 \%$ ) and $89.3 \%$ (95\% CI 78.3-91.9\%) respectively.

In the study of Amatya et al. (2018), LUS was positive with pneumonia, demonstrating a sensitivity of $91 \%$. CXR was positive patients with pneumonia, yielding a sensitivity of $73 \%$. The sensitivity of ultrasound was significantly better than CXR. Specificity of LUS and CXR were similar at $61 \%$ and $50 \%$ respectively. The positive predictive value of lung ultrasound was $85 \%$ and CXR was $78 \%$. The negative predictive value of LUS was $73 \%$, while CXR was $43 \%$. The positive likelihood ratio for diagnosing pneumonia with LUS was 2.34 , while the negative likelihood ratio was 0.15 . CXR had a positive and negative likelihood ratio of 1.45 and 0.55 respectively.

Haggag et al., (2019) found that LUS had a sensitivity of $100 \%$ and accuracy of 95\% in pneumonia diagnosis compared to the sensitivity and accuracy of $72.3 \%$ and $81 \%$, respectively for CXR.

The limitations of our study included a relatively short follow up period and the diagnostic efficacy of US for other complications as lung abscesses and necrotic lesions was not assessed due to lack of such patients.

\section{CONCLUSION}

LUS could be considered as a good diagnostic and follow up tool when pneumonia is suspected, while CT chest may be reserved in cases where ultrasound is technically difficult or when there is discrepancy with clinical findings.

\section{REFERENCES}

1. Agmy G and Ahmed Y (2013): Role of transthoracic sonography in diagnosis and follow up of community acquired pneumonia 
in emergency department. Eur Respir J., 42(Suppl 57):228-32.

2. Alkhayat KF and Alam-Eldeen MH (2014): Value of chest ultrasound in diagnosis of community acquired pneumonia. Egyp J Chest Diseases and Tuberculosis, 63:1047-51.

3. Amatya Y, Rupp J, Russell FM, Saunders J, Bales B and House DR (2018): Diagnostic use of lung ultrasound compared to chest radiograph for suspected pneumonia in a resource-limited setting. International Journal of Emergency Medicine, 11:8-13.

4. Andrea T, Lauetani F, Nouvenne A, Mori G, Chiussi $G$ and Maggio $M$ (2016): Lung ultrasound and chest X-ray for detecting pneumonia in an acute geriatric ward. Medicine (Baltimore), 95 (27): e4153:1-7.

5. Blaivas M (2012): Lung ultrasound in evaluation of pneumonia. J Ultrasound Med., 31:823-6.

6. Caiulo VA, Gargani L, Caiulo S, Fisicaro A, Moramarco F and Latini G (2013): Lung ultrasound characteristics of communityacquired pneumonia in hospitalized children. Pediatr Pulmonol., 48:280-7.

7. Cortellaro F, Colombo S, Coen D and Duca PG (2012): Lung ultrasound is an accurate diagnostic tool for the diagnosis of pneumonia in the emergency department. Emerg Med J., 29 (1):19-23.

8. Fahmy $H$ and Kinawy $S$ (2018): The Accuracy of Lung Ultrasonography versus Chest Radiography for Diagnosis and Followup of Pneumonia in Critically Ill Patients. Med. J. Cairo Univ., 86 (7):3799-3805.

9. Haggag YI, Mashhour K, Ahmed K, Samir $N$ and Radwan W (2019): Effectiveness of Lung Ultrasound in Comparison with Chest XRay in Diagnosis of Lung Consolidation. Open
Access Maced J Med Sci., 15; 7(15):24572461.

10. Lichtenstein DA (2009): Ultrasound examination of the lungs in the intensive care unit. Pediatric Crit. Care Medi., 10 (6): 693-8.

11. Moghawri MWS, Mansour W, Lakouz KA and Hussein RMH (2017): Role of chest ultrasonography in the diagnosis and follow-up of community-acquired pneumonia at Zagazig University Hospitals. Egypt J Bronchol., 11:29-35.

12. Parlamento S, Copetti $R$ and DiBartolomeo $S$ (2009): Evaluation of lung ultrasound for the diagnosis of pneumonia in the ED. Am J Emerg Med., 27(4):379-384.

13. Rennis KD, Anaz M, Krishnakumar EV, Thomas V and Robert AP (2017): Role of chest ultrasonography in the prognostic evaluation of patients with community acquired pneumonia: a prospective study. Int $\mathbf{J}$ Res Med Sci., 5:25-30.

14. Saraya S and EI Bakry R (2017): Ultrasound: Can it replace CT in the evaluation of pneumonia in pediatric age group? The Egyptian Journal of Radiology and Nuclear Medicine, 48:687-694.

15. Yang $X$, Yinghua $Y$, Shaobin $W$, Wen $L$ and Huahao S (2016): Effectiveness of lung ultrasonography for diagnosis of pneumonia in adults: A systematic review and meta-analysis. J. Thorac. Dis., 8 (10): 2822-31. 
دور الموجات الصوتية علي الرئة في تشخيص ومتابعة

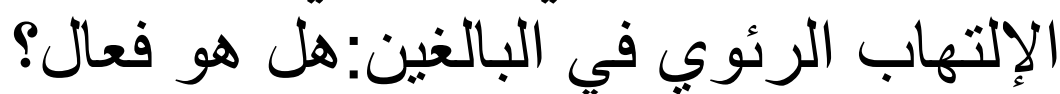

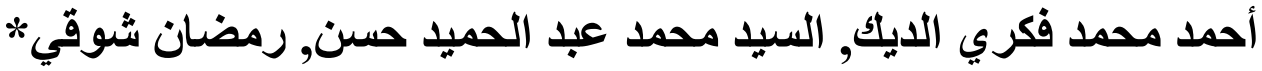
قسمي الأثعة التثخيصية والأمراض الصدرية*، كلية الطب، جامعة الأزهر (فرع دمياط) E-mail: deekscan@yahoo.com

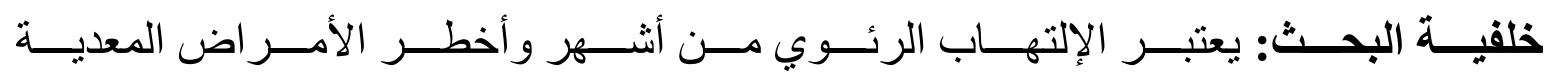

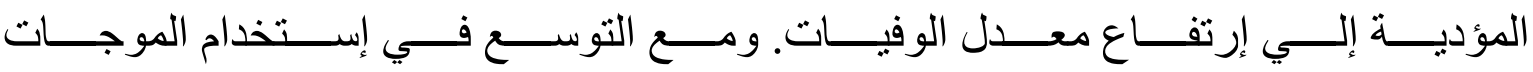

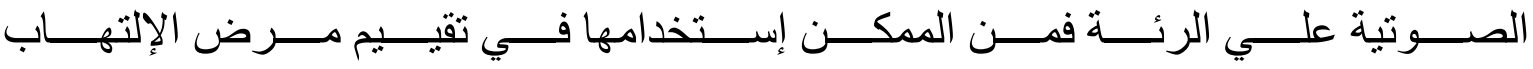

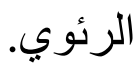

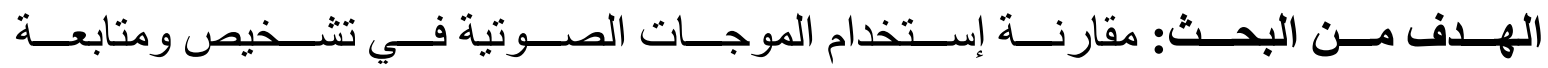
الإلتهاب الرئوي في البالغين مقارنة بالوسائل النتخيصية المعتمدة.

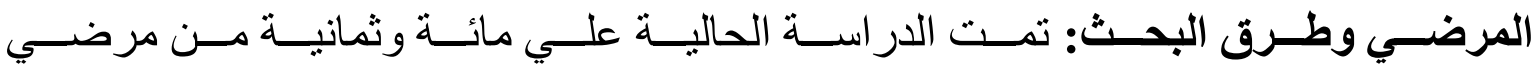

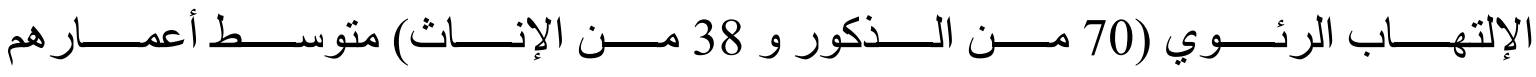

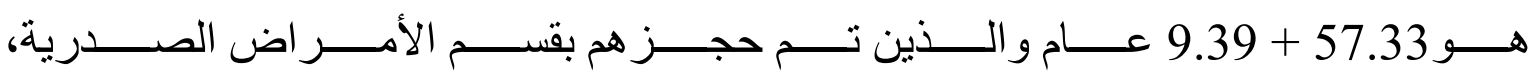
مستثشفي الأزهر الجامعي بدمياط في الفترة من مارس وحتي نوفمبر 2019.

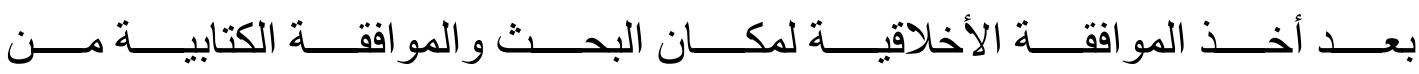

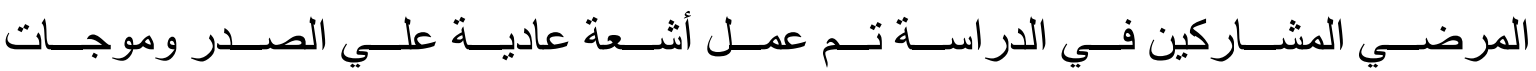

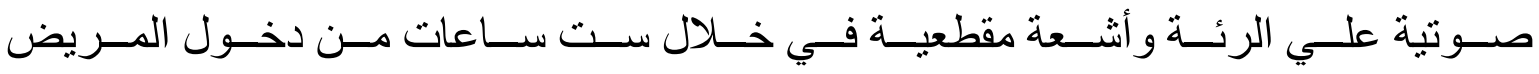

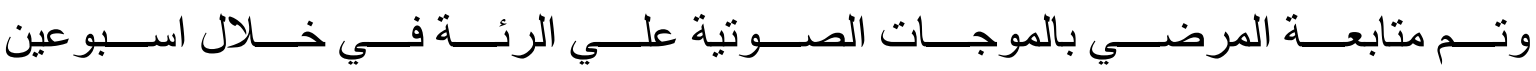

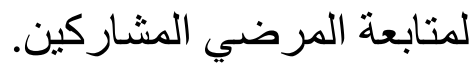

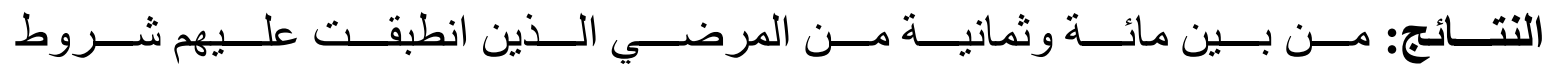

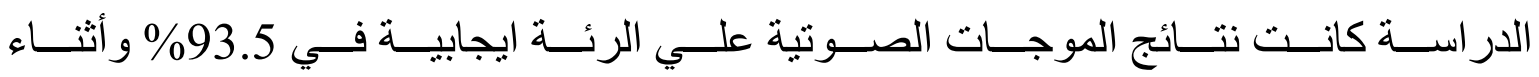

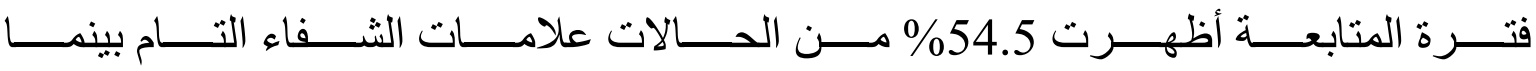

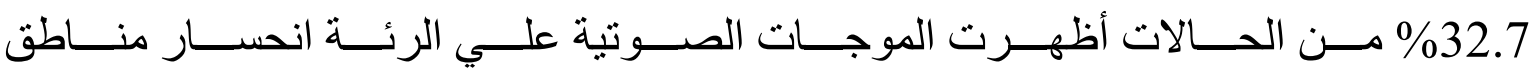




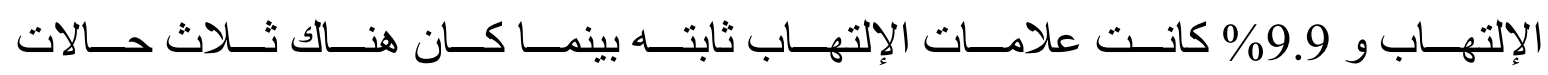

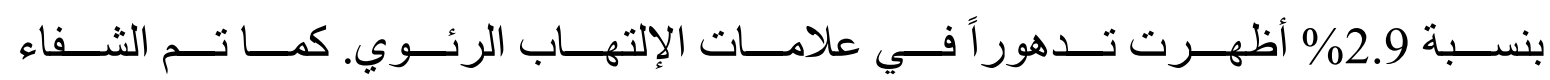
التام في 90\% من حالات الإنصباب الجنبي المصاحب للإلتهاب الرئوي .

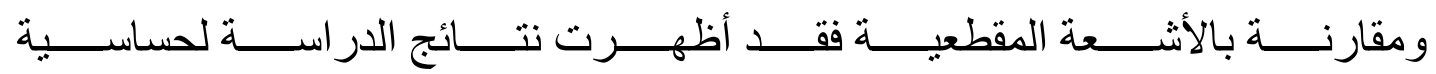

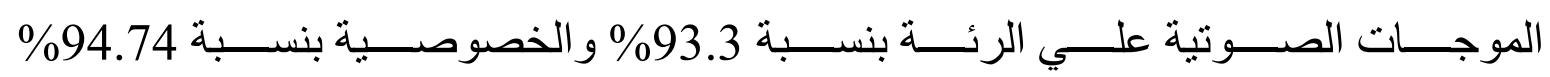
ودقة النتخيص بنسبة 93.91\%.

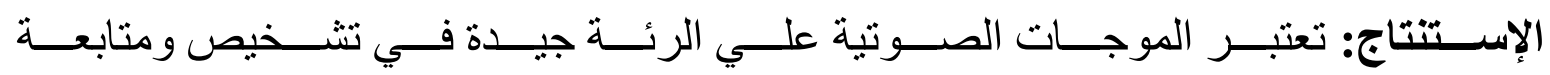

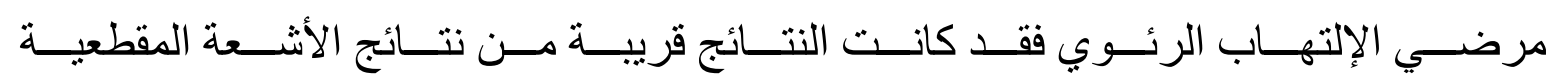
مع إنخفاض التكلفة وتجنب التعرض لمخاطر الأشعة.

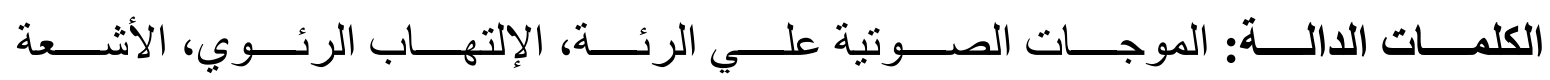
المقطعية. 